\title{
INFLUENCE OF TOOL-WORKPIECE INTERFACE ON SURFACE INTEGRITY AFTER TURNING
}

This paper deals with influence of flank wear on decomposition of cutting force in turning roll bearing steel. The decomposition is carried out to evaluate the components of cutting force in the tool-chip as well as tool-workpiece interface. The decomposition enables evaluation of the shear and normal forces in the both interfaces. The results of experiments and the following calculations show that while conditions for chip separation in the tool-chip interface stay nearly untouched due to progressive development of flank wear, mechanical load of machined surface associated with components of cutting forces progressively increases with flank wear. The paper also reports about influence of flank wear on surface integrity expressed in such parameters as residual stresses, surface topography, structure transformation and microhardness.

Keywords: Cutting force, decomposition, flank wear, turning, surface integrity.

\section{Introduction and theoretical background}

Surface integrity can be expressed in a variety of parameters and features. Machined surface is a product of tool-workpiece interface. Due to a certain radius of cutting edge a certain volume of material undergoes the cutting edge. Thickness of the layer undergoing the cutting edge depends mainly on the cutting edge radius and this layer is well known as a minimum chip thickness. This layer can be reported as a minimum cutting depth needed for chip separation [1]. Surface integrity in turning expressed in terms of surface roughness depends mainly on the tool geometry and cutting conditions (mainly feed). On the other hand, surface integrity expressed in such terms as residual stresses, structure and hardness alteration depends mainly on mechanical and thermal load of machined surface in tool-workpiece interface. Flank wear (VB) takes the significant role since it remarkably affects toolworkpiece interface. Flank wear progressively increases within the cutting time and undergoing material is exposed to the high mechanical and thermal load. While mechanical alteration affects mainly the near-surface region, thermal load can penetrate deeply beneath the surface as the flank wear is progressively developed.

Many studies were reported about influence of flank wear on surface integrity expressed in such terms as stress state, microhardness profile and structure [2, 3 and 4]. As it was mentioned, these aspects of surface integrity are affected mainly by mechanical and thermal load. On the other hand, it should be also pointed that heat and temperature are only the different features associated with the energy consumption derived from forces needed for cutting process. Being so, information about components of cutting force attributed to the pure tool-workpiece interface is essential for analysis of surface integrity. It is well known that decomposition of cutting force can be carried out in a variety of models. Conventional decomposition provided by Merchant [5] ignores existence of cutting edge radius or flank wear. Decomposition of cutting force is associated with pure processes needed for chip separation in the tool-chip interface.

When the cutting edge radius or flank wear are considered, decomposition of cutting force is considered as the decoupling of two basic components. First one is attributed to the tool rake and corresponds with chip separation while the second component is associated with the tool-workpiece interface as Fig. 1 depicts. $F_{y}$ component is associated with the energy needed for chip separation in the tool-chip interface while $F_{a}$ is component affecting mainly quality of machined surface. Cutting force can be then expressed as follows:

$$
\bar{F}=\overline{F_{\gamma}}+\overline{F_{\alpha}}(N)
$$

Decomposition of cutting force, in relation to tool wear and cutting edge rounding, was discussed by many authors [6 - 8]. It was found that flank wear $(V B)$ affects produced surface integrity expressed in a variety of features such as stress state, surface roughness, microhardness and structure as well as topography of machined surface [3, 4, 9 - 11].

\footnotetext{
* Jaroslav Dubec, Miroslav Neslusan, Anna Micietova, Maria Cillikova

Department of Machining and Manufacturing Engineering, University of Zilina, Faculty of Mechanical Engineering, Slovakia E-mail: jaroslav.dubec@fstroj.uniza.sk
} 


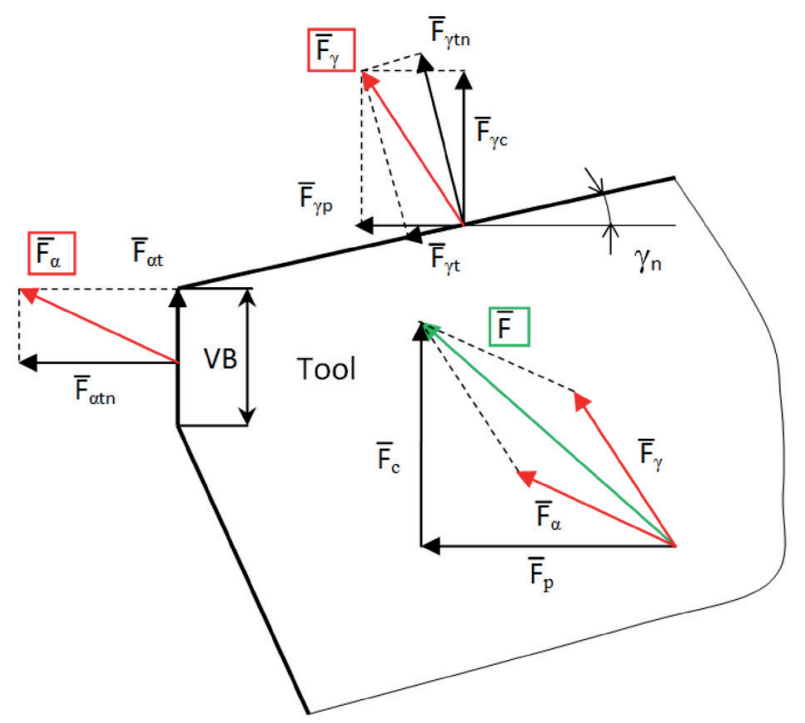

Fig. 1 Decomposition of cutting force in relation to tool wear [1]

For this reason this paper reports about experimental technique for decomposition of cutting force associated with equation (1). The paper is focused on the turning of bearing steel with cutting insert of variable flank wear (see Fig. 2) to discuss why the cutting force is increasing with the progressive flank wear $V B$ and how the components $F_{v}$ and $F_{a}$ contribute to cutting force increase.

\section{Experimental conditions}

Experiments were conducted on annealed bearing steel $100 \mathrm{Cr} 6$ by the use of cutting insert SNMG 120408E-M $\left(\alpha_{0}=9^{\circ}\right.$, $\left.\gamma_{0}=-6^{\circ}, r_{n}=80 \mu \mathrm{m}\right)$. Components of cutting force were measured by KISTLER 9265B dynamometer (lathe SUI 40) under the following cutting conditions:

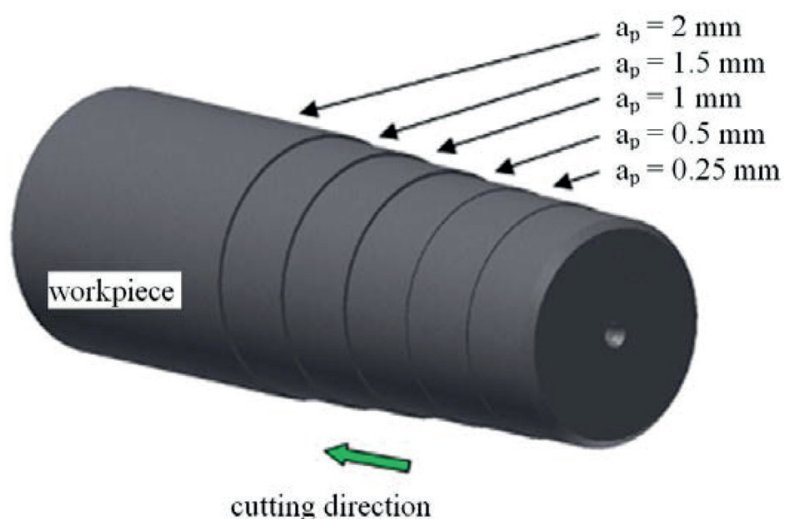

Fig. 3 Workpiece of variable cutting depth adapted for measurement

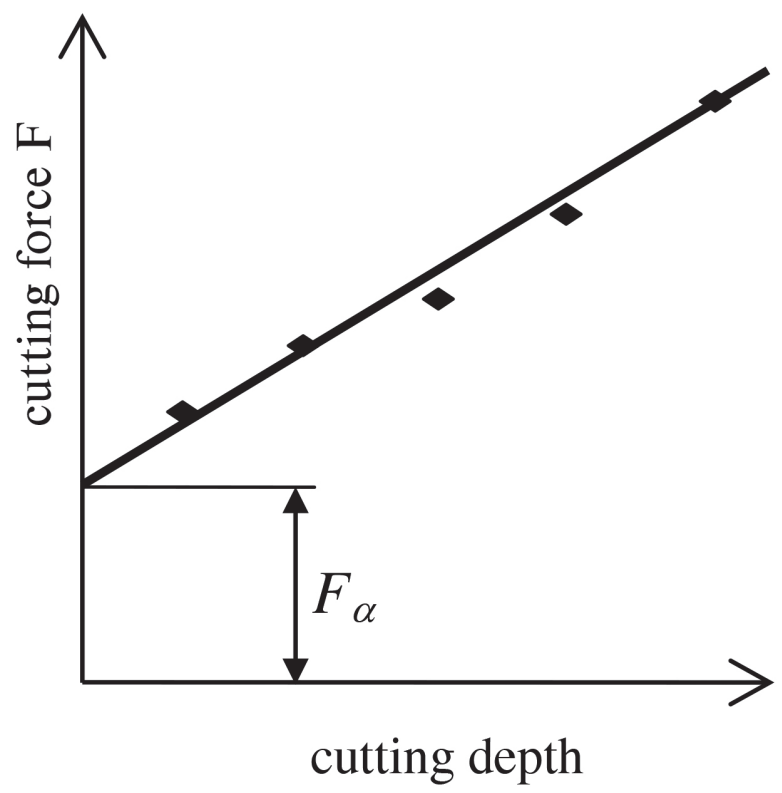

Fig. 4 Graphical illustration of $F_{a}$ evaluation

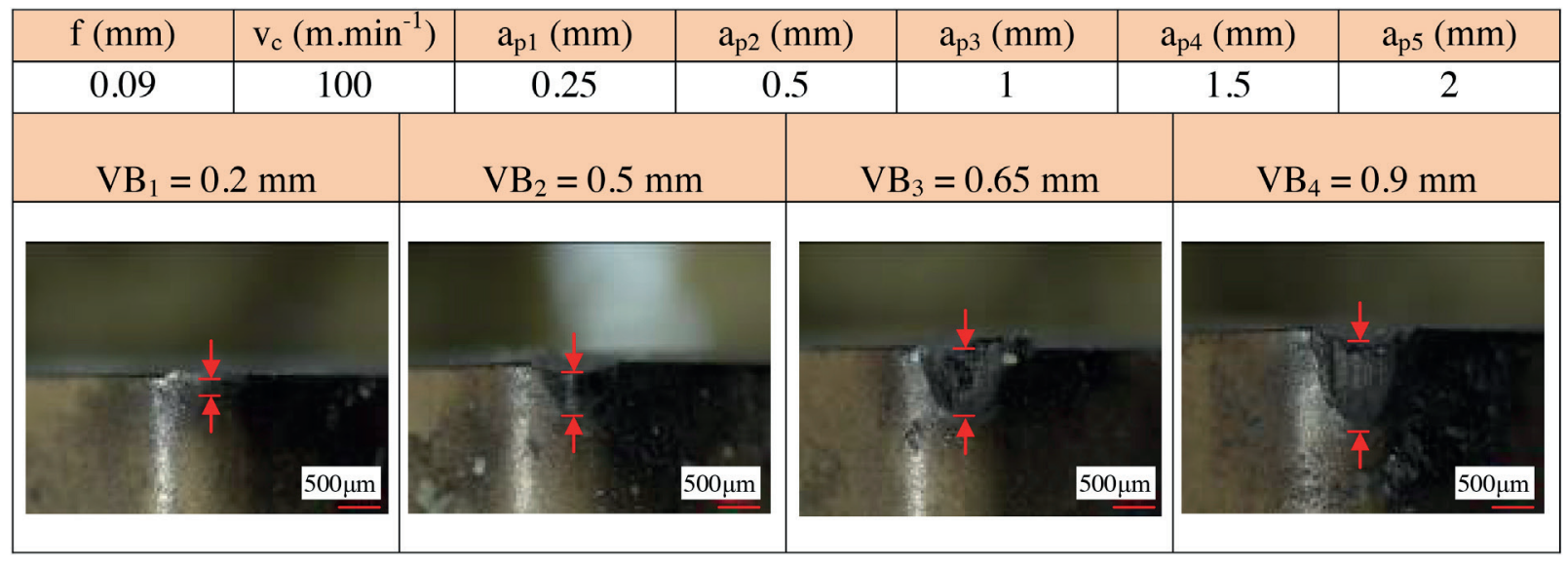

Fig. 2 Cutting insert of the different VB applied for cutting test, microscope AxioCam ER c5c 
To analyze the influence of flank wear $V B$ on $F_{y}$ and $F_{a}$ components inserts of variable $V B$ were prepared during the preliminary turning, see Fig. 2. Workpieces illustrated in Fig. 3 were pre-prepared to obtain the variable cutting depth and to analyze relation between the measured components and cutting depth. Separation of $F_{a}$ component is based on calculation of trend line as a relationship between measured cutting force components and the corresponding cutting depths. $F_{a}$ can be found as a point on the trend line in position where cutting depth is equal to zero, see Fig. 4. Residual stresses were measured by mechanical method based on electrolytic etching of a machined surface (home made system). Vickers microhardness measurement was conducted by Hanneman 300 micro-hardness tester by applying force $300 \mathrm{~N}$ for 10 seconds.

\section{Results of experiments}

Figure 5 shows the relationship where $F_{p}$ and $F_{c}$ are plotted against the different cutting depths and flank wears. Figures 5, 6 and 7 also show that flank wear affects mainly thrust force component $F_{p}$ since the thrust force component is mostly attributed to the processes in tool-workpiece interface while only the moderate increase of $F_{c}$ component is mostly associated with chip separation in the tool-chip interface.

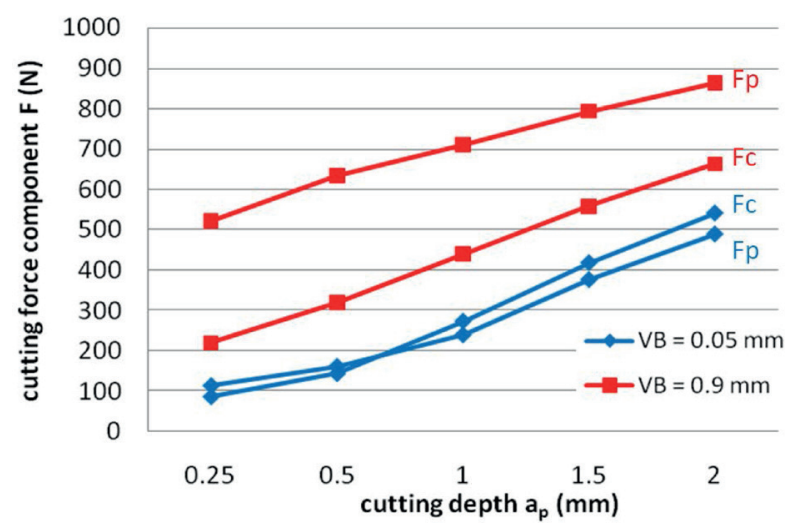

Fig. 5 Influence of $a_{p}$ on components of cutting force, $V B=0.05 \mathrm{~mm}-$ sharp insert, $V B=0.9 \mathrm{~mm}$

Being so, increasing area of flank wear contributes mainly to the more pronounced increase of thrust force. It should be also mentioned that except for flank wear which is progressively increasing within the cutting time, tool wear in form of a crater is also developed on the rake face of the insert. However, the crater alters mainly the rake angle of the insert when initial negative geometry (negative rake angle for sharp insert) becomes more positive along with increasing flank wear $V B$. Results of force measurements indicate that the effect of flank wear dominates over the effect of crater developed on the rake of the insert since cutting force components progressively increase.

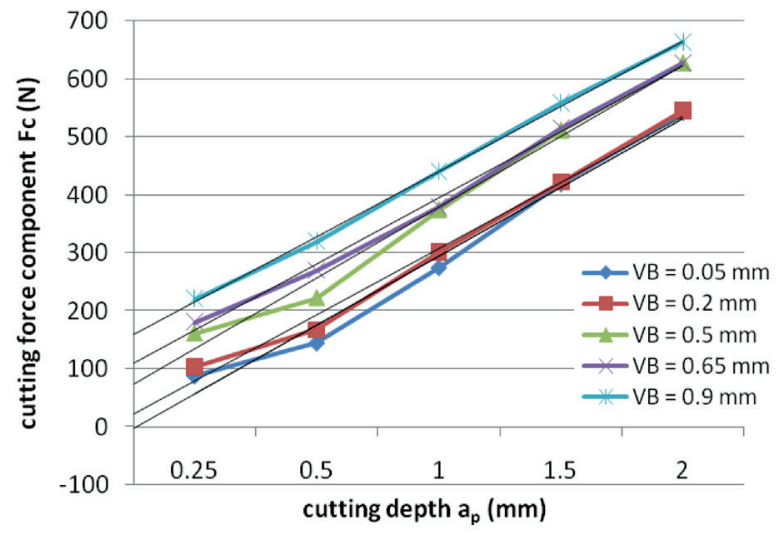

Fig. 6 Influence of cutting depth and flank wear on $F_{c}$

Results of decomposition, based on evaluation of intersection points (in position when cutting depth is equal to zero), enable evaluation of components associated with the tool-chip as well as tool-workpiece interface (and the corresponding shear and normal components). Figure 8 a depicts that $F_{\gamma}$ depends mainly on cutting depth and the corresponding removal rates.

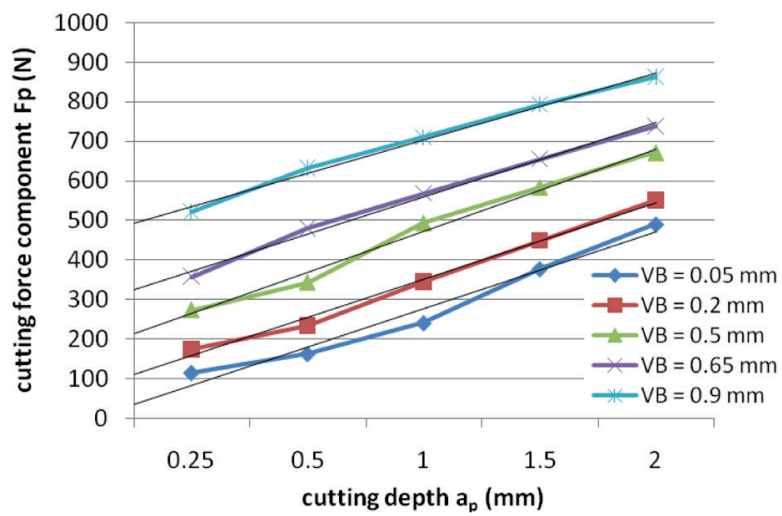

Fig. 7 Influence of cutting depth and flank wear on thrust force $F_{p}$

On the other hand, this component stays nearly untouched when flank wear is progressively developed. In other words, flank wear does not contribute to the processes of chip separation in the tool-chip interface and affects mainly mechanical load of surface produced in the tool-workpiece interface. Figure $8 \mathrm{~b}$ illustrates that $F_{a}$ stays nearly untouched at the different cutting depths but abruptly increases with flank wear $V B$. 


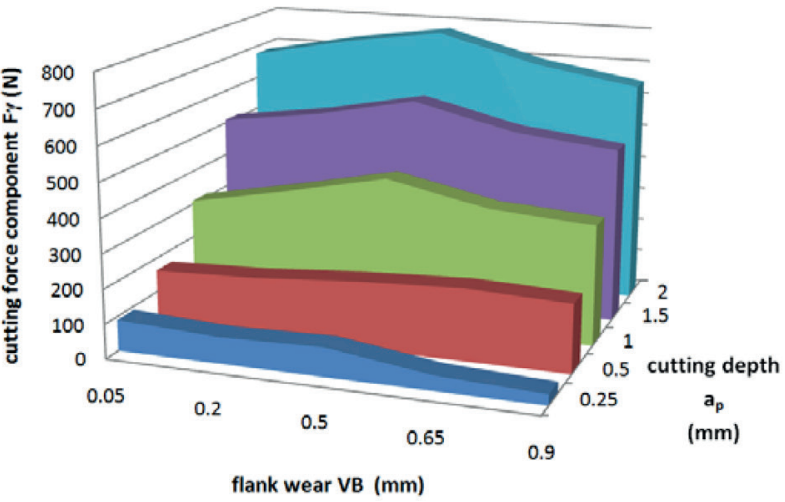

a)

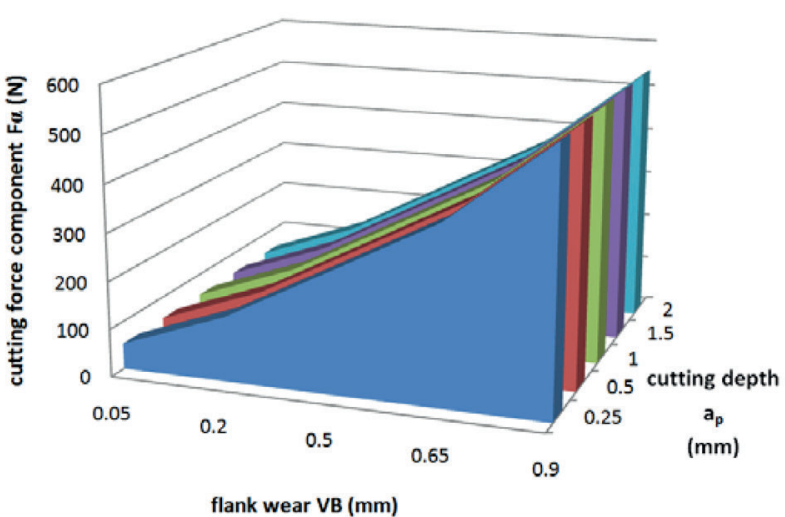

b)

Fig. 8 Influence of tool wear and cutting depth on a) $F_{\gamma}$-face, b) $F_{a}$-flank

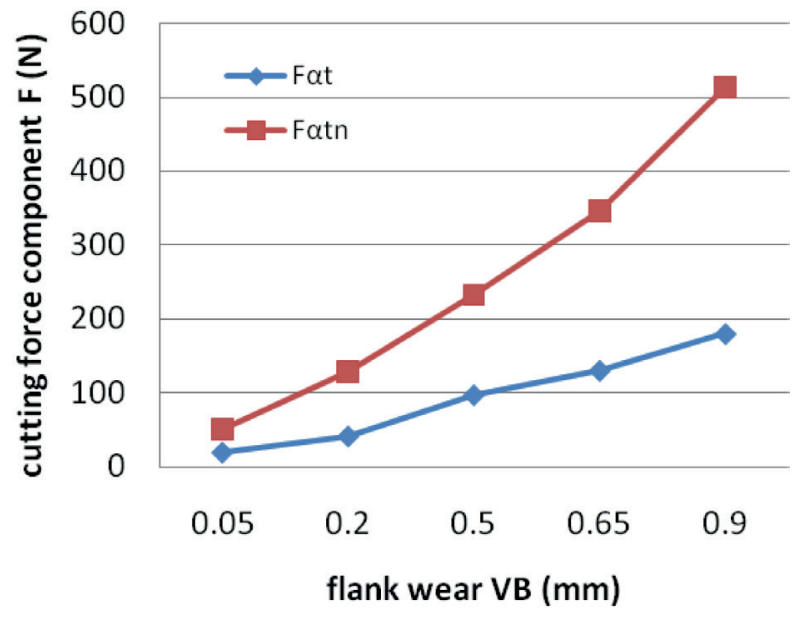

Fig. 9 Influence of flank wear on $F_{a t}$ and $F_{\text {atn }}$ $\left(F_{a t}\right.$ extracted from Fig. 6 and $F_{\text {atn }}$ extracted from Fig. 7 as the values at zero cutting depth)

Figure 9 shows that normal force component in the toolworkpiece interface dominates over the shear component in correspondence with more pronounced increase of thrust force along with increasing flank wear $V B$.

\section{Surface integrity}

This chapter discusses influence of flank wear on surface integrity expressed in many terms. Tool wear changes dimension and shape of a cutting edge. For this reason, temperature and stress distribution ahead a cutting edge is transformed, which in turns corresponds with variable mechanical and thermal load of a machined surface as well as the appearance of a separated chip. While processes of chip separation are mainly affected by progressive transformations of tool geometry on the tool rake, processes in the tool-workpiece interface affect mainly surface integrity.
It is commonly accepted that mechanical and thermal loads are closely connected since the energy consumption needed for cutting process is converted into heat. Total heat generated in the cutting zone can be expressed as follows:

$Q=F_{c} \cdot v_{c} \quad\left(\mathrm{~J} \cdot \mathrm{min}^{-1}\right)$

As it was reported, cutting force can be decoupled as the component needed for chip separation $F_{\gamma}$ and the component associated with chip-tool interface $F_{a}$. The average mechanical load of machined surface can be expressed via $F_{a t}$ and $F_{a t n}$ components. On the other hand, a certain idea about thermal load on machined surface can be obtained when the heat generated in the tool-workpiece interface is calculated as follows:

$Q_{f}=F_{a t} v_{c} \quad\left(\mathrm{~J} \mathrm{~min}^{-1}\right)$

Thus increasing $F_{a t}$ component directly corresponds with the heat generated in the tool-workpiece interface which in turn corresponds with elevated temperatures when the flank wear $V B$ is more developed.

It is well known that residual stresses are induced by cutting process. Compressive stresses are obtained when mechanical load dominates whereas elevated temperatures in the tool-workpiece interface cause tensile stresses. It is also well known that machined surface usually undergoes severe plastic deformation as well as intensive thermal load. Being so, when no structure transformation occurs, residual stress state of machined surface is a mixture of both effects. Figure 10 illustrates distribution of residual stresses beneath the surface. Compressive stresses dominate in the near-surface region after turning. Thin surface layer undergoes the severe plastic deformation due to a certain cutting edge radius as well as flank wear. Figure 10 also shows that the compressive stresses in the near-surface region increases along with flank wear -160 MPa can be found after turning with the sharp cutting insert. When the flank wear $V B$ becomes more developed the maximum of compressive stresses increases in 


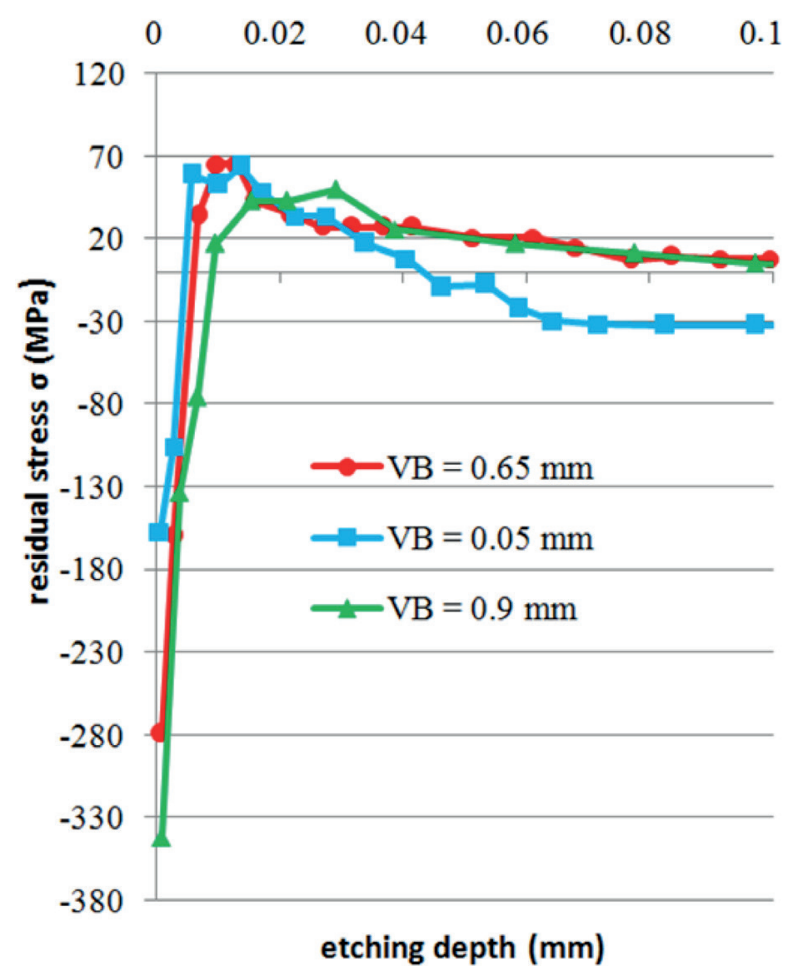

Fig. 10 Depth profile of residual stresses obtained after turning with insert of variable $V B$

correspondence with increasing $F_{a t}$ and $F_{a t n}$ components (-280 $\mathrm{MPa}$ was obtained for $V B=0.65 \mathrm{~mm}$ and $-345 \mathrm{MPa}$ for $V B=0.9$ $\mathrm{mm})$. Figure 10 also shows that thickness of near-surface region containing compressive stresses increases when the flank wear $V B$ is developed. Furthermore, Fig. 10 illustrates the differences in the stress state in the sub-surface region. Sub-surface layers contain tensile stresses of nearly the same magnitude. On the other hand, tensile stresses extend deeper beneath the surface

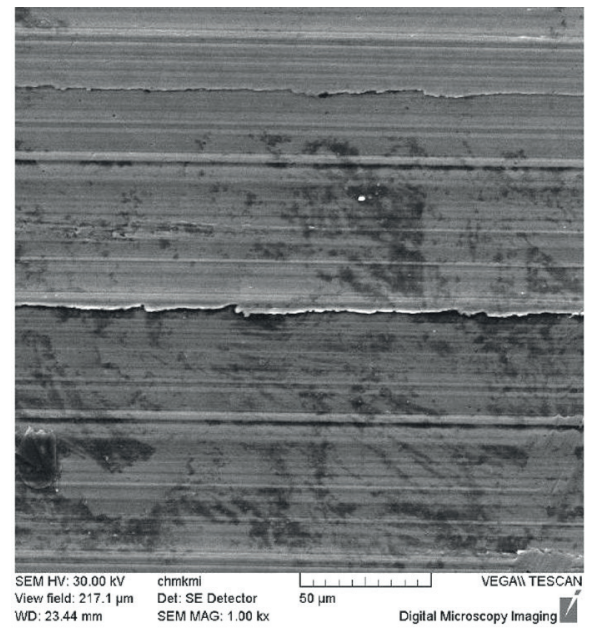

a) sharp tool, $V B=0.05 \mathrm{~mm}$

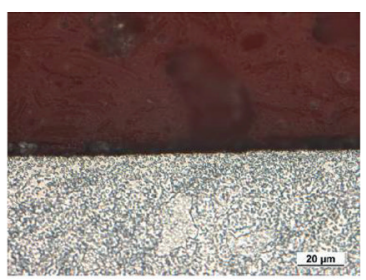

a) sharp tool, $V B=0.05 \mathrm{~mm}$

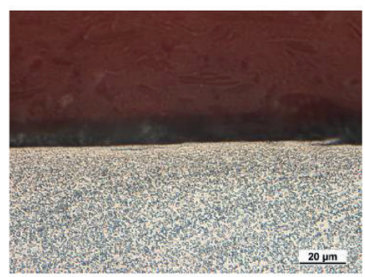

b) $V B=0.65 \mathrm{~mm}$

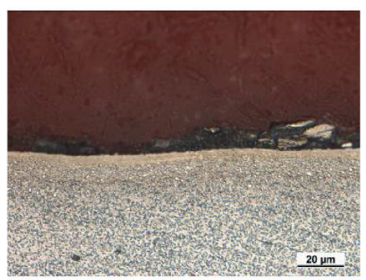

c) $V B=0.9 \mathrm{~mm}$

Fig. 11 Micrographs of machined surface, optical microscopy,

cross sections

at high flank wear than those obtained for low $V B$. Thickness of thermally affected zone for higher $V B$ (where tensile stresses dominate) exhibits twofold increase in thickness than that produced by sharp insert.

Observation of surfaces obtained for all $V B$ reveals that structure alterations occur as soon as $V B$ exceeds $0.5 \mathrm{~mm}$, see Fig. 11. While significant texture with very thin and discontinuous

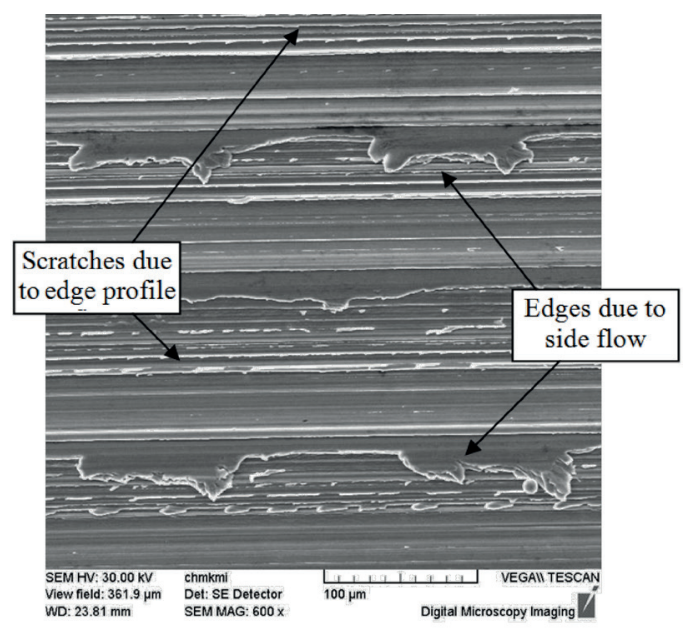

b) $V B=0.65 \mathrm{~mm}$

Fig. 12 Micrographs of machined surface, REM 
transformed layer can be found on the surface produced with insert of $V B=0.65 \mathrm{~mm}$ (Fig. 11b), more pronounced structure transformation occurs when flank wear reaches $0.9 \mathrm{~mm}$ (Fig. 11c). Surface texturing is associated with intensification of plastic deformation of undergoing layer together with longer time interval within the produced surface is exposed to severe plastic deformation at elevated temperatures. It was previously discussed [4] that cutting forces and temperature in the cutting zone gradually increase up to the $V B=0.65 \mathrm{~mm}$ with a certain fall when visible structure transformations on the produced surface can be observed $(V B=0.9 \mathrm{~mm})$.

Intensity and character of plastic deformation during chip separation can be also evidenced by topography of produced surface as Fig. 12 depicts. Machined surface is quite smooth with the typical pattern produced by turning at low flank wear, see Fig. 12a. On the other hand, intensive side flow during severe plastic deformation in the tool-workpiece interface results in formation of the edges on the machined surface as Fig. 12b illustrates. Moreover, the furrowed profile of abraded cutting edge produces surface with many scratches since a cutting edge profiles copies on machined surface. Thus flank wear strongly affects surface roughness of machined surface.

Hardness of steels is explained in concept of high dislocation density as the carbide precipitation takes minor role. Being so, measured microhardness profiles correlate with microstructure observations and considered density of dislocation cells.

While moderate increase of surface microhardness is attributed to the surfaces without visible structure transformations (about $400 \mathrm{HVm}$ for near-surface region, bulk microhardness is $300 \mathrm{HVm}$ ), steep increase of the near surface hardness (685 $\mathrm{HVm}$ ) correlates with hardened structure undergoing the severe plastic deformation and containing the high dislocation density.

\section{Conclusions and final comments}

Additional investigations [9] show that deformations after heat treatment depend on surface integrity and the corresponding flank wear $V B$. Being so, detailed understanding of these aspects can help us to suppress these deformations and reduce the corresponding stocks, time and cost savings as a significant aspects of improvements in production [12]. Roll bearings are usually heat treated to produce the surface of high hardness and the corresponding wear and fatigue resistance. Despite the surface produced before heat treatment is removed after heat treatment by the following grinding of hard turning, surface integrity expressed in many terms (mainly stress state, microhardness and structure) strongly affects deformation of parts (especially of parts made of thin wall or expressed in other words the parts of high wall thickness to diameter ratios).

\section{Acknowledgement}

This article was edited under the financial support of VEGA project No. 1/0223/11 and 1/0097/12.

\section{References}

[1] NESLUSAN, M., CILLIKOVA, M.: Cutting Theory, EDIS: University of Zilina, 2007

[2] BRANDT, D.: Randzonenbeeinflussung beim Hartdrehen, Dissertation, Hannover, 1995

[3] WANG, J.Y., LIU, C.R.: The effect of Tool Flank Wear on the Heat Transfer, Thermal Damage and Cutting Mechanics in Finishing Hard Turning, CIRP Annals 48/1/1999

[4] DUBEC, J., NESLUSAN, M.: Multiparametric Analysis of Surface after Turning through Barkhausen Noise in Relation to Tool Wear, MM Science J., July 2012,

[5] MERCHANT, M. E.: Mechanics of the Metal Cutting Process, J. of Applied Physics, 16/1945

[6] BENO, J.: Theory of Metal Cutting, Kosice: Vienala, 1999

[7] ENDRES, W. J., DEVOOR, R. E., KAPOOR, S. G.: Dual Mechanism Approach to the Prediction of Machining Forces. J. of engineering for industry, ASME, 1995

[8] EE, K. C., BALAJI, A. K., LI, P. X., JAWAHIR, I. S.: Force Decomposition Model for Tool-Wear in Turning with Grooved Cutting Tools, Wear, 249/2001

[9] DUBEC, J., NeSluSAN, M., CILliKOVA, M., MICIETOVA, A.: Magnetic Evaluation of Residual Stresses and Structure Transformations Induced in Soft Steel after Turning, Nanomaterials and nanotechnology meeting, 1/2013

[10] KARPUSCHEWSKI, B., SCHMIDT, K., PRILUKOVA, J., BENO, J., MANKOVA, I., HIEU, N. T.: Influence of Tool Edge Preparation on Performance of Ceramic Tool Inserts when Hard Turning, J. of Materials Processing Technology, 213, 2013, $1978-1988$

[11] CZAN, A., TILLOVA, E., SEMCER, J., PILC, J.: Surface and Subsurface Residual Stresses after Machining and their Analysis by s-ray Diffraction, Communications - Scientific Letters of the University of Zilina, No. 2, 2013, ISSN 1335-4205

[12] MICIETA, B., STOLLMANN. V.: Design and Improvement of Production Processes, DAAAM Intern. Scientific Book 2009, ISSN 1726-9687, ISBN 978-3-901509-69-8. 